\title{
Does time to endoscopic sinus surgery impact outcomes in chronic rhinosinusitis? Retrospective analysis using the UK clinical practice research data*
}

\author{
C. Hopkins' ${ }^{1}$ P. Andrews' ${ }^{2}$, C.E. Holy ${ }^{3}$ \\ ' Guy's Hospital, ENT Department, London, UK \\ ${ }^{2}$ Royal National Throat, Nose and Ear Hospital, London, UK \\ ${ }^{3}$ Acclarent Inc, Menlo Park, CA, USA
}

\author{
Rhinology 53: 18-24, 2015 \\ DOI:10.4193/Rhino14.077 \\ *Received for publication: \\ March 17, 2014
}

Accepted: September 4, 2014

\begin{abstract}
Background: Patients with chronic rhinosinusitis (CRS) refractory to medical management may elect endoscopic sinus surgery (ESS). Recent data showed that clinical outcomes of patients treated earlier outperformed those of patients treated later in the disease continuum. In this study, CRS-related healthcare utilisation of patients treated early versus late was analysed using the UKbased Clinical Practice Research Database.
\end{abstract}

Methods: Two cohorts ("Early Cohort": ESS within 12 months of first CRS diagnosis, versus "Late Cohort": $\geq 5$ years from diagnosis to ESS) were matched for age, gender, asthma, polyposis. Healthcare needs related to CRS were analysed post-operatively for 5 years.

Results: Patients in the Late cohort used significantly more CRS-related care than patients in the Early cohort visits and 0.54 prescriptions per patient per year. A sub-analysis of patients with and without asthma indicated that patients in the Late cohort without asthma had healthcare needs equivalent to patients in the Early cohort with asthma.

Conclusion: Delayed surgical intervention for CRS is associated with greater post-operative healthcare needs than ESS within 12 months of first CRS diagnosis.

Key words: paranasal sinuses, observational study, sinusitis, surgical endoscopy, health resources

\section{Introduction}

Chronic rhinosinusitis (CRS) is a condition that is estimated to affect $11 \%$ of the UK population ${ }^{(1)}$, yet little is known of the natural history and disease progression, as highlighted in a recent 2012 study $^{(2)}$. It is not documented whether early surgical treatment of CRS offers better long-term outcomes than treatments provided after many years of CRS diagnosis.

A recent sub-analysis of the UK National Comparative Audit of Surgery for Nasal Polyposis and Chronic Rhinosinusitis compared the clinical outcomes of CRS following endoscopic sinus surgery of patients treated surgically within 1) less than 1 year; 2) 1 to 5 years or 3) more than 5 years of medical management of CRS disease. This study showed that patients treated earlier in the history of the disease (i.e., within 12 month of first diagnosis) experienced significantly greater symptomatic benefits from the surgical intervention than patients with longer CRS medical histories ${ }^{(3)}$.

In this study, we evaluated whether the findings from the UK national Comparative Audit would be generalisable to the broad UK population and over a large number of years. We therefore analysed a patient cohort from the Clinical Practice Research Data (CPRD), previously known as GPRD, for healthcare utilisation post-surgery, based on time of first diagnosis. This database includes longitudinal medical records of patients registered with primary care practices across the UK, and as of 2012, included 8.5\% of the UK population, representing England, Northern Ireland, Scotland and Wales. Data in the database extends as far back as 1987 and linkages to hospitalisation data have been added sinc6e April 1997. In March 2013, as many as 12.6 million patients were deemed of research quality, with $55 \%$ having 
complete hospitalisation data ${ }^{(4)}$. CPRD has been considered the gold standard for database research and has led to more than 800 peer-reviewed publications ${ }^{(5)}$.

\section{Materials and Methods}

The protocol for this study was approved by to CPRD's Independent Scientific Advisory Committee (approval \# 13_184R).

\section{Cohort identification}

A cohort of patients undergoing an endoscopic sinus surgery, defined by a procedure code (OPCS-4) of E13.X-E17.X after 01/01/2000 was identified. Patient records were further evaluated and patients with low-quality records or non-continuous data coverage were eliminated. For the remaining cohort, a number of analyses were performed to identify an "Early" and a "Late" cohort, as described below and shown in Figure 1.

As a preliminary step, the complete observation period available for each patient was identified. This observation period corresponds to the period in-between the first and last data collection date for each patient's GP practice.

To determine the date of first diagnosis, the medical history for all patients with sinus surgery in the database was analysed and all read codes associated with a definite diagnosis of sinusitis or polyps were manually queried. The date of the medical visits when these codes were first utilised was identified for all patients. The time frame between a patient's first diagnosis of CRS and date of first validated, available medical entries in the database (i.e., first "recorded" date) was calculated and defined as the "washout" period. Patients with a washout period less than 12 months were eliminated as the date of first diagnosis could not reasonably be estimated.

The time frame was then established from the date of first diagnosis to the date of surgery. Based on that time window, patients were grouped in the following sub-cohorts: 1) First diagnosis to surgery within 12 months ("Early Cohort"); 2) First

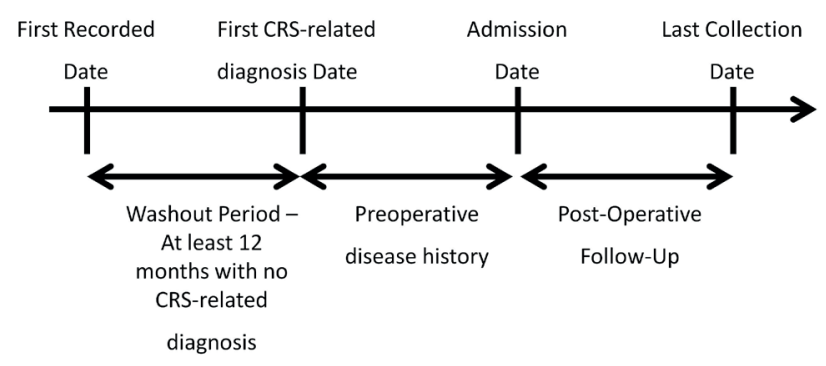

Figure 1. Information captured for all patients, to determine length of data available in the database, time of first diagnosis vs. first available data and post-operative follow-up period. diagnosis to surgery between 1.01 year and 4.99 years ("Mid Cohort") and 3) First diagnosis to surgery greater than 4.99 years ("Late Cohort"). As the study was designed to understand differences in healthcare utilisation patterns between Early vs. Late cohort, all analyses described herein focus on the Early versus Late cohorts only.

The time frame from surgery to last data collection was analysed for all patients and defined as the "postoperative follow-up period". Patients with a post-operative period less than 6 months were eliminated from the database.

The number of available patient-years was then analysed for both cohorts. For this analysis, the available follow-up for each patient was defined for Years 1 through 5 (Year 1 starting the day after surgery and ending on day 366 post-operative, Year 5 starting on day 1466 and ending on day 1831 post-operative.)

\section{Matched cohort sub-analysis}

In an attempt to decrease the potential impact of confounders, Early and Late cohorts were matched for age at time of surgery, gender and prevalence of asthma and polyps at time of surgery. The matching of cohorts was performed using the PROC SURVEYSELECT procedure from SAS, as defined elsewhere ${ }^{(6)}$. Each asthma-polyp-age-gender combination was used as a separate stratum.

\section{Medical history}

The medical read codes identified in the entire medical history were further reviewed to identify all codes associated with diagnoses, visits and treatments of sinusitis and related disease. This second list of codes, broader than that defined to strictly identify first date of CRS diagnoses, was used to extract all visits and prescriptions relevant to CRS. All visits were analysed on the basis of time from surgery, starting on day 1 post-operative up to 5 years post-surgery.

\section{Prescription history}

All prescriptions linked to the medical visits from the query described above were identified. Prescriptions were grouped by British National Formulary (BNF) chapters and reviewed manually to ensure all included prescriptions were targeted to treatment of CRS disease. Prescriptions were then analysed on the basis of time to surgery as explained above, and categorized by main drug category.

\section{Impact of patients with asthma}

A sub-analysis was performed, in which the frequency of procedures and drug use as described above was further evaluated for the following separate cohorts: 1) Early cohort without Asthma; 2) Early cohort with Asthma; 3) Late cohort without asthma and 4) Late cohort with asthma. 
Table 1. Demographic and key comorbidities of patients in the Early and Late cohort, before matching.

\begin{tabular}{|lcc|}
\hline N & $\begin{array}{c}\text { Early Cohort - } \\
\text { Prior to } \\
\text { Matching }\end{array}$ & $\begin{array}{c}\text { Late Cohort - } \\
\text { Prior to } \\
\text { Matching }\end{array}$ \\
\hline $\begin{array}{l}\text { Percentage Female } \\
\text { Age } \\
\text { Mean (95\% Cl) }\end{array}$ & 39\% & 1,074 \\
\hline $\begin{array}{l}\text { Percentage patients with } \\
\text { asthma at time of surgery }\end{array}$ & $47.9(47.0-48.2)$ & $52.5(51.7-53.3)$ \\
\hline $\begin{array}{l}\text { Percentage patients with } \\
\text { polyps at time of surgery }\end{array}$ & $24.0 \%$ & $30.5 \%$ \\
\hline $\begin{array}{l}\text { Percentage patients with both, } \\
\text { asthma and polyps, at time of } \\
\text { surgery }\end{array}$ & 48.0\% & \\
\hline $\begin{array}{l}\text { Number of years from First } \\
\text { Diagnosis to Surgery } \\
\text { Mean (95\% Cl) }\end{array}$ & $13.7 \%$ & $40.8 \%$ \\
\hline
\end{tabular}

Table 1 represents the key demographic and comorbid conditions of patients in the preliminary cohorts, prior to matching. Interestingly, the Late cohort had more cases of asthma and fewer cases of polyposis than the Early cohort.

\section{Statistical analyses}

Descriptive statistics and analyses (means, standard deviations [SD], and confidence intervals) were calculated using SAS Enterprise Guide 4.3 (SAS Institute, Cary, NC, USA). Continuous data were presented as means with confidence intervals. Healthcare utilisation data points (healthcare utilisation per patient per year, frequency of prescriptions and types of prescriptions per patient per year) being non-normally distributed, statistical comparisons of these non-parametric data points were conducted using Wilcoxon Rank Sum tests.

\section{Results}

Prior to the matching process, there were 1,460 patients in the Early cohort and 1,074 patients in the Late cohort. Table 1 summarizes key demographics for these cohorts. Interestingly, at time of surgery, there were significantly more patients with asthma (30.5\% vs. $24.0 \%)$ and significantly fewer patients with polyps $(40.8 \%$ vs. $48.01 \%)$ in the Late vs. Early cohort. The ratio of patients with both, asthma and polyps, increased from $13.7 \%$ in the Early cohort to $16.6 \%$ in the Late cohort.

To better understand the difference in asthma prevalence between the pre-matched cohorts, an analysis was conducted to determine the percentage of patients with diagnosed asthma

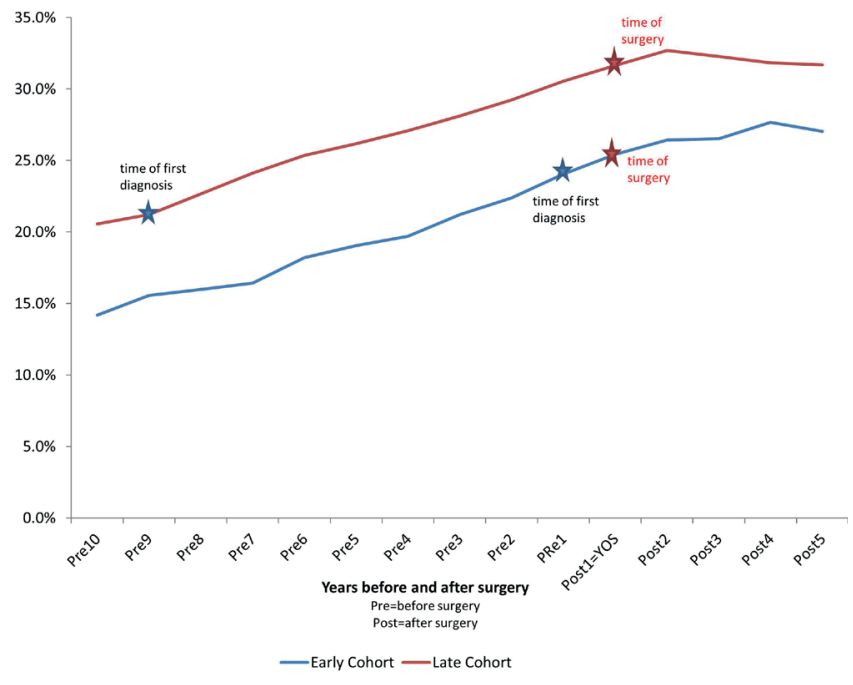

Figure 2. Percentage of patients in the pre-matched cohort with asthma, at various time points relative to time of surgery. At time of first diagnosis (blue star), both cohorts had comparable rates of asthma. Rate of new asthma diagnoses declined for both cohorts after surgery.

at time points starting 10 years before surgery up to the end of the 5-year post-operative period. Figure 2 shows the percentage of patients with asthma at all time points. At time of first diagnosis, both cohorts had a comparable rate of asthma $(21.2 \%$ for the Late cohort, $24.1 \%$ in the Early cohort). After surgery, the rate of new asthma diagnosis slowed down for both cohorts.

To reduce the potential confounding effect of age, gender, asthma and polyps on CRS-related healthcare utilisation outcomes, each cohort was then matched for these 4 variables. The final cohorts comprised each 764 patients at time of surgery (46.3\% female, average age: 51.0, 95\%Cl: 50.1 - 52.0, proportion of patients with asthma $=23.9 \%$, proportion of patients with

Table 2. Patient-years for each post-operative time interval in the matched cohorts.

\begin{tabular}{|lccccc|}
\hline & Year 1 & Year 2 & Year 3 & Year 4 & Year 5 \\
\hline Early Cohort & 755.8 & 704.4 & 518.7 & 610.4 & 441.2 \\
\hline Late Cohort & 758.1 & 710.8 & 499.3 & 602.9 & 407.1 \\
\hline
\end{tabular}

Table 2 represents the total number of patient-year for each post-operative time interval. All patients included in the study had at minimum 6 months of post-operative history in the database. However, beyond the first 6 months, follow-up periods varied. For the "Year 5 " follow-up period, there were therefore 880.2 patient-years in the Early Cohort and 877.9 patient-years in the Late Cohort. 


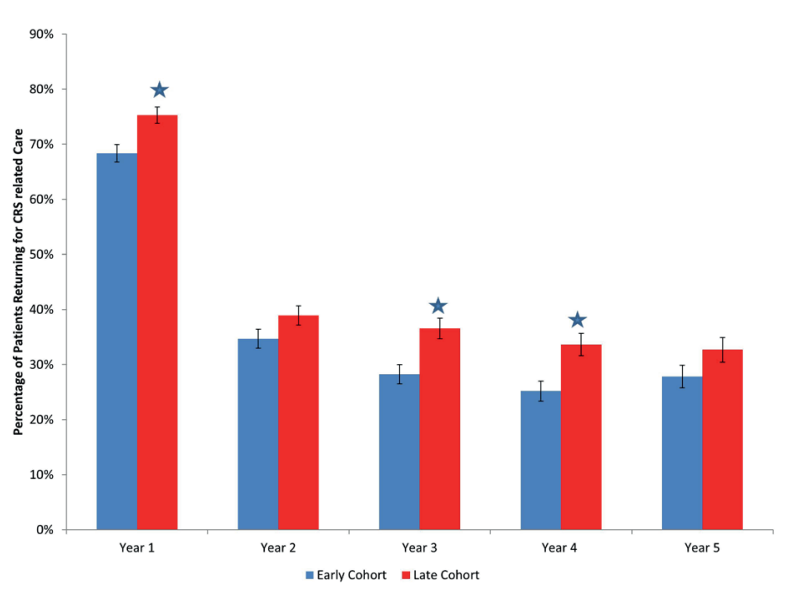

Figure 3. Average percentage of patients returning for post-operative healthcare related to CRS at each post-operative time points. Significant differences between Early and Late cohort are shown with *.

polyps $=40.8 \%)$. Duration from first diagnosis to surgery was 0.51 years (95\%Cl: $0.49-0.53)$ and 9.2 years $(95 \% \mathrm{Cl}: 9.0-9.5)$ for the Early and Late cohorts, respectively.

The number of patient-year available at each follow-up period is shown in Table 2. Both cohorts had comparable patient-years at all follow-up time periods, with approximately 400 patient-years still available to each cohort at 5 years post-operative.

Immediately post-operative, a majority of patients returned for at least one visit related to CRS (68.4\% of the Early and $75.3 \%$ of the Late cohort). From years 2 to 5 post-operative, these ratio dropped to approximately one third of the cohorts returning for care. Figure 3 shows the percentage of returning patients at all time points. For all time points, the percentage of patients returning for care in the Late cohort exceeded that in the Early cohort. These differences were significant on years 1, 3 and 4 . The average number of medical visits over the 5 -year period for CRS reached 0.85 visits per patient per year ( $95 \% \mathrm{Cl}: 0.79-0.91)$ in the Early cohort and 1.06 visits per patient per year $(95 \% \mathrm{Cl}$ : $1.00-1.13)$ in the Late cohort. This difference was significant ( $p<0.0001)$. The number of visits per year per patient is shown in Figure 4. In all years, there were more visits per patient in the Late vs Early cohort. The difference in frequency of visits was significant on years 3 and 4 post-operative.

The number of prescriptions strictly related to CRS visits and CRS condition was also significantly greater for patients in the Late cohort vs Early cohort, with an average number of prescriptions per patient per year in Early Cohort of 0.36 (95\%Cl: 0.33 - 0.40) and an average number of prescriptions per patient per year in Late Cohort: 0.54 (95\% Cl: 0.48 - 0.60). This difference was significant $(p<0.0001)$. The overall frequency of prescription per cohort per year is shown in Figure 5. The difference in prescrip-



Figure 4. Average frequency of medical visits and consultations postoperatively by patient-year. Significant difference between Early and Late cohort are shown with *.

tion frequency was significant on years 3 and 4 post-operative.

A further analysis of prescription by prescription type indicated that for all prescription categories evaluated, the frequency of use for patients in the Early cohort were lower than that of patients in the Late cohort for all drug types except for leukotriene modifiers, which were rarely used (Figure 6).

Reoperation rates were analysed for both, the Early and Late cohort. Over the 5 year period, the reoperation rate reached $11.86 \%$ in the Early cohort ( $95 \% \mathrm{Cl}: 9.41 \%-14.32 \%$ ) and $11.52 \%$ in the Late cohort $(9.06 \%-13.97 \%)$. There was no difference in reoperation rates.

A sub-analysis looking at: Group 1) Early Patients without asthma, Group 2) Early Patients with asthma, Group 3) Late patients without asthma and Group 4) Late patients with asthma was conducted. Figure 7a shows the average number of CRS-related medical visits and Figure $7 \mathrm{~b}$ shows the average number of prescriptions per patient per year over the entire 5 year study, for all groups. Both visits and prescriptions were significantly lower for Group 1) Early Patients without asthma versus all other Groups. Group 2 (Early Patients with asthma) and Group 3 (Late patients without asthma) had comparable healthcare use, whereas Group 4 (Late patients with asthma) had greatest healthcare use.

\section{Discussion}

In this study, CRS-related healthcare utilisation following sinus surgery for diagnosed chronic rhinosinusitis was evaluated, using the CPRD database. Patients were grouped based on time from first CRS diagnosis to surgery, with patients in the Early cohort treated within 12 months of first diagnosis and patients in the Late cohort, being treated after 5 years of diagnosed disease. To reduce impact of confounding disease, cohorts were 


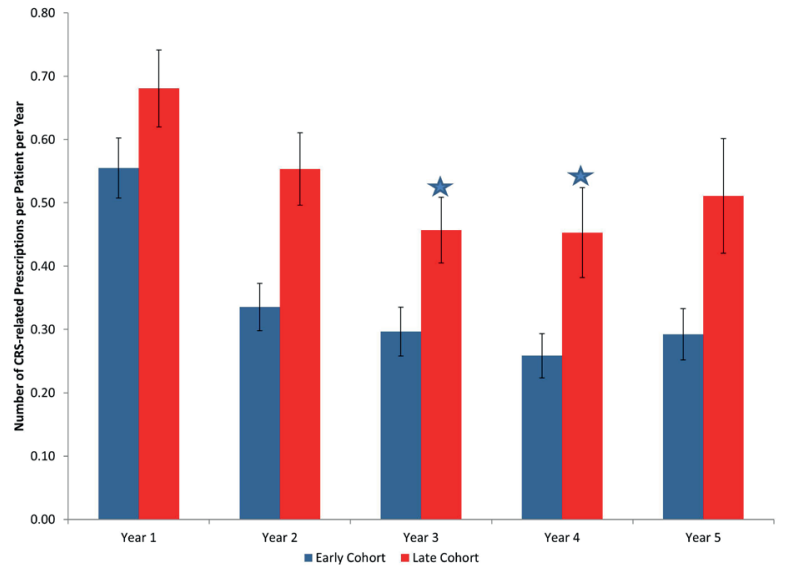

Figure 5. Average frequency of prescriptions by patient-year. Significant differences between Early and Late cohort are shown with *.

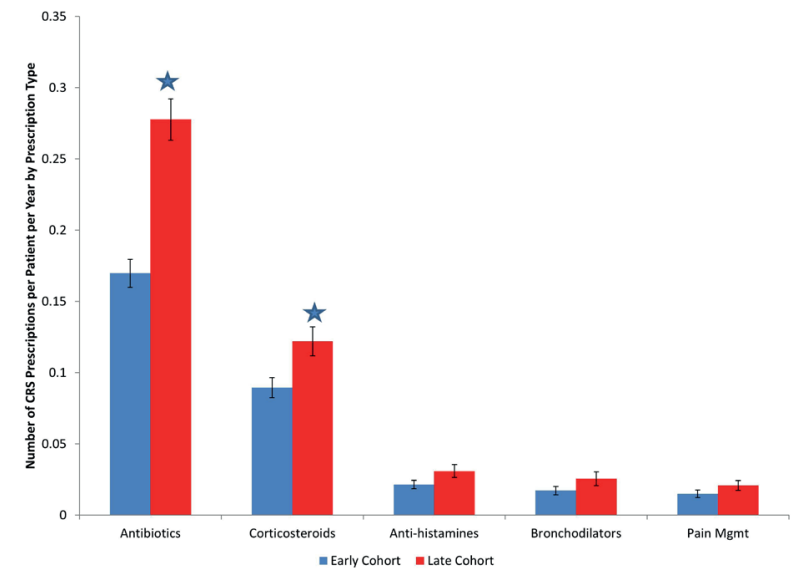

Figure 6. Average frequency of prescriptions by prescription type for patients in Early vs Late cohort. Significant differences are shown with *. matched for presence of asthma and polyps at time of surgery. Results indicated fewer patients in the Early cohort returned for CRS-related care in all 5 years following surgery versus patients in the Late cohort. Overall number of visits and prescriptions were significantly lower in the Early cohort versus the Late cohort.

The reason why delaying surgical intervention potentially lowers prognosis can only be hypothesised as no definite study has been published on the natural history of CRS and its long term impacts when left untreated. Mucosal remodeling has been well documented in patients with CRS, specifically leading to a thickening of the basement membrane, an increase in submucosal acinar cells and loss of cilia ${ }^{(7)}$. The severity of osteitis has been shown to be significantly correlated to duration of symptoms ${ }^{(8)}$. It can therefore be hypothesised that a long-term, unresolved CRS condition may lead to profound tissue damages that may be more difficult to correct than if treated early on. A sub-analysis looking at the impact of asthma on CRS-related care further revealed that patients in the Late cohort without asthma had overall needs for CRS visits and prescriptions similar to those of patients in the Early cohort with asthma. Patients in the Early cohort without asthma had the lowest CRS healthcare needs, whereas patients in the late cohort with asthma and the highest healthcare needs of all groups. This finding suggests that delayed intervention in patients without asthma potentially lowers the outcomes of surgical intervention such that these patients end up with healthcare needs for CRS comparable to those of patients treated early with asthma.

The increased proportion of asthma patients in the pre-matching Late cohort is an interesting finding, also observed in the prior clinical study by Hopkins et al ${ }^{(3)}$. Whereas no guideline or other documented record exists suggesting that surgical care should be delayed for CRS patients with asthma, both this database study and the Audit analysis opens a question on whether delayed surgical intervention may be associated with greater risks of asthma. Further research is required to further understand this issue, as improving management of CRS also improves lower respiratory function and providing adequate and timely treatment to patients with - or susceptible to - asthma is essential.

In our study, we also observed a greater proportion of polyp patients in the initial Early group. We hypothesize that the need for surgery may be perceived to be greater in CRSwNP, and therefore these patients are likely to be referred to secondary
(A)

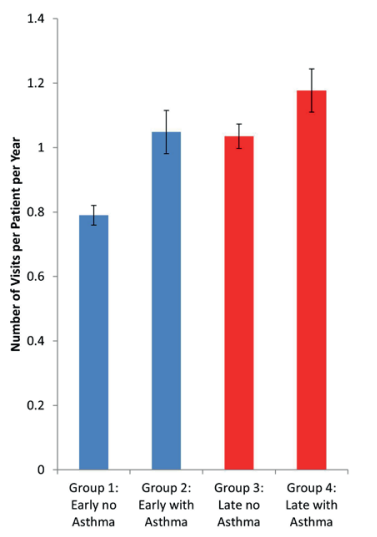

(B)

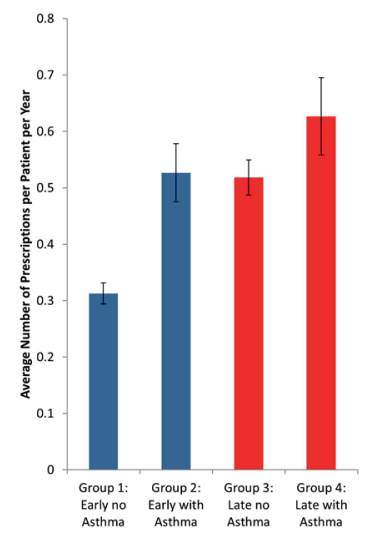

Figure 7. Healthcare utilisation per patient-year over 5-year study period for patients in both cohort, based on co-diagnosis of asthma. (A) number of visits; (B) Average count of prescriptions. 
care faster than patients without polyps, and also submitted to surgery at an earlier stage. In contrast, CRSsNP poses more of a diagnostic challenge, and symptoms may be attributed to other conditions. These patients with CRsNP therefore undergo a more prolonged trial of medical therapy before surgery is considered. To eliminate any possible confounder, however, our final cohort was matched for prevalence of polyps.

A limitation of this study includes the fact that the data was obtained retrospectively from a research database not specifically designed to study sinus disease. As a result, all limitations inherent to database research apply herein, such as possible incomplete data collection from participating physicians, underreporting of specific diagnoses, incorrect coding, loss of information on visits to non-participating physicians or centres. It is also important to note that all diagnoses were made by primary care physicians and were not verified by CTs. In addition, mild polyps may not have been diagnosed. However, it is anticipated that these potential limitations apply equally to all groups and therefore general trends across groups would still be meaningful of actual healthcare utilisation. We also captured all prescriptions related to visits attributed to CRS. Our list of medications included some not recommended for the treatment of CRS according to latest consensus guidance ${ }^{(9)}$ - however, we believe this reflects current prescribing patterns in primary care, and this potential bias applied equally to both cohorts.

Another limitation is the fact that the washout period (time from first recorded information to first diagnosis of CRS) was only 12 months. It is possible that some patients may have had a year without symptoms at time of enrollment and then a new diagnosis after the 12-month washout. These patients would have been included in the Early Cohort even though disease may have been present prior to enrollment in CPRD.

In our study, the benefits of surgical intervention for CRS treatment - defined by reduction in healthcare needs - seem to decrease with increased time to surgery. We have previously shown in an independent patient cohort that symptomatic benefit decreased with increased time to surgery ${ }^{(3)}$. Similar hypotheses have already been put forward by Benninger ${ }^{(8)}$ and others ${ }^{(3,11-13)}$ and highlight the need for effective and timely resolution of CRS symptoms. Current European Guidelines recommended that surgery be considered after 12 weeks of failed medical management ${ }^{(9)}$. This recommendation, if applied, would ensure that all patients refractory to medical management get treated within at most 12 months of unresolved CRS diagnosis and may experience greatest benefits from surgical treatment.

Surgical intervention has also been shown to be cost effective. A cost burden analysis of CRS showed a reduction in the costs related to their CRS treatment post surgery ${ }^{(14)}$. Medication, consultation and investigation costs all significantly reduced in patients with CRS post surgery both in the first and second years following surgery. However, the cost of the surgical procedure remains significant, and there is a need to maximise the effectiveness of the intervention.

There is little in the published literature on optimal timing for surgical intervention. Smith et al. ${ }^{(15-16)}$ evaluated a group of patients with CRS undergoing either surgical intervention or continued medical management. After 3 months post-operative, $37 \%$ of patients in the continued medical management had failed and crossed over to the surgical arm. With 3 distinct groups: the surgical arm, the continued medical management arm and the cross-over arm, Smith et al. reported that patients in the surgical intervention group had statistically significantly better outcomes than patients in both other groups at 6 months post-operative - despite the only real difference between the surgical arm and the cross-over arm being merely a 3-months delay in surgical intervention. This study thus supports our finding that once medical therapy has been found to be unsuccessful, avoiding unnecessary delay before proceeding to surgical intervention may improve outcomes.

Beyond direct healthcare costs associated with treating ongoing symptoms, chronic rhinosinusitis represents a significant societal cost, accounting for a reduction in productivity of $38 \%$ in patients affected by the disease ${ }^{(16)}$. Delays in achieving adequate symptom control are likely to increase indirect costs and may offset any apparent savings in the direct costs of surgery.

Current austerity measures in most countries with nationalised healthcare systems such as the UK are likely to increase delays in referral and limit our ability to treat patients with CRS in a timely fashion. Attempts to reduce health-care expenditure by restricting access to secondary care should be carefully considered, as such measures may have negative and lasting impact on patients' ability to experience meaningful improvements from CRS symptoms, and increase health-care expenditure in the longer term.

\section{Acknowlegdement}

License to the CPRD database was funded by Acclarent and Johnson \& Johnson.

\section{Authorship contribution}

$\mathrm{CH}$ and $\mathrm{CEH}$ : study design, data analysis and preparation of manuscript.

PA: study design and editorial input to manuscript.

\section{Conflicts of Interest}

None 


\section{References}

1. Hastan D, Fokkens WJ, Bachert C, et al. Chronic rhinosinusitis in Europe--an underestimated disease. A GA(2)LEN study. Allergy. 2011; 66: 1216-1223.

2. Sedaghat AR, Bhattacharyya N Radiographic density profiles link frontal and anterior ethmoid sinuses behavior in chronic rhinosinusitis. Int Forum Allergy Rhinol. 2012; 2: 496-500.

3. Hopkins C, Rimmer J, Lund VJ. Does time to endoscopic sinus surgery impact outcomes in Chronic Rhinosinusitis? Prospective findings from the National Comparative Audit of Surgery for Nasal Polyposis and Chronic Rhinosinusitis. Rhinology. 2015, 53: 10-17.

4. CPRD. The UK General Practice Research Database. A Powerful e-Clinical Research Tool. Clin Res Focus 2002; 13: 48-50.

5. CPRD at www.cprd.com, accessed May 29, 2013.

6. Diseker R. Simplified Matched Case-Control Sampling using PROC SURVEYSELECT. In: SUGI 29 Proceedings. Montreal: www2.sas. com; 2004

7. Pawankar R, Nonaka M. Inflammatory mechanisms and remodeling in chronic rhinosinusitis and nasal polyps. Curr Allergy Asthma Rep. 2007; 7: 202-208.
8. Georgalas C, Videler W, Freling N, Fokkens W. Global Osteitis Scoring Scale and chronic rhinosinusitis: a marker of revision surgery. Clin Otolaryngol. 2010; 5: 455-461.

9. Fokkens WJ, Lund VJ, Mullol J, et al. European Position Paper on Rhinosinusitis and Nasal Polyps 2012. Rhinol Suppl. 2012; 3: 1-298.

10. Benninger MS, Ferguson BJ, Hadley JA, et al. Adult chronic rhinosinusitis: definitions, diagnosis, epidemiology, and pathophysiology. Otolaryngol - Head Neck Surg 2003; 129: S1-32.

11. Penttila M, Rautiainen M, Pukander J, et al. Functional vs. radical maxillary surgery. Failures after functional endoscopic sinus surgery. Acta Otolaryngol Suppl. 1997; 529: 173-176.

12. Senior B and Kennedy D. Long-term results of FESS. Laryngoscope. 1998; 108: 151-156.

13. Osguthorpe JD. Surgical outcomes in rhinosinusitis: what we know. Otolaryngol Head Neck Surg. 1999; 120: 451-452.

14. Bhattacharyya N, Orlandi RR, Grebner J, et al. Cost of burden of chronic rhinosinusitis: A claims-based study. Otolaryngol - Head Neck Surg 2011; 144: 440-445.

15. Smith TL, Kern R, Palmer JN, et al. Medical therapy vs surgery for chronic rhinosinusitis: a prospective, multi-institutional study with 1-year follow-up. Int Forum Allergy Rhinol. 2013; 3: 4-9.

16. Smith TL, Kern RC, Palmer JN, et al. Medical therapy vs surgery for chronic rhinosinusitis: a prospective, multi-institutional study. Int Forum Allergy Rhinol. 2011; 1: 235-241.

17. Stankiewicz J, Tami T, Truitt T, et al. Impact of chronic rhinosinusitis on work productivity through one-year follow-up after balloon dilation of the ethmoid infundibulum. Int Forum Allergy Rhinol. 2011; 1: 38-45.

Claire Hopkins, BM BCh MA (Oxon)

DM FRCS (ORL-HNS)

ENT Dept.

Guy's Hospital

London SE1 9RT

United Kingdom

Tel: +44-20-7188 2215

Fax: +44-20-7188 2206

E-mail address:

clairehopkins@yahoo.com

\section{ADVERTISEMENT}

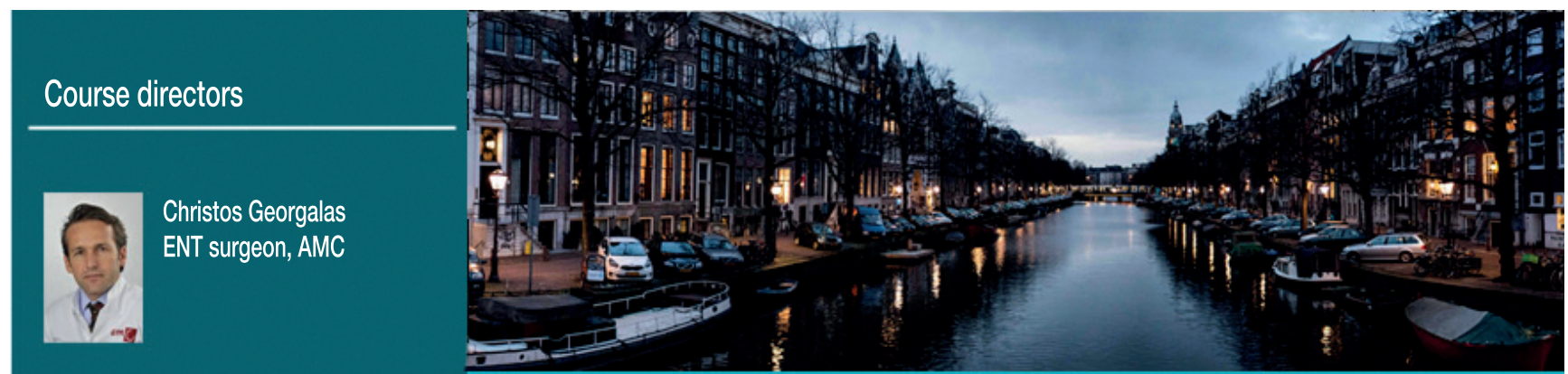

Wouter van Furth Neurosurgeon, AMC

\section{Faculty}

Shahzada Ahmed (UK) ENT Isam Alobid (E) ENT Paolo Castelnuovo (I) ENT Ricardo Carrau (USA) ENT lacopo Dallan () ENT Daniel Kelly (USA) Neurosurgeon Michael Mokry (D) Neurosurgeon Daniel Prevedello (USA) Neurosurgeon Anshul Sama (UK) ENT

\section{2nd Endoscopic Skull Base Masterclass \& Symposium}

Symposium:

Nuances in pituitary surgery

- Full course (incl. dissections) | 1400 euro

- Combined team ENT/Neurosurgeon | 1200 euro

- Lectures only $\mid 700$ euro

- Step by step "How I do It" demonstrations

$-1: 3$ faculty to participant ratio

- Fresh Frozen specimens

- Neuro-Navigation and High Speed Endoscopic Drills - Memory stick with HD dissections included

\section{7 - 18 - 19 June 2015 Amsterdam}

www.skullbasecourse.nl

Course secretariat: M. van Huiden Academic Medical Center, University of Amsterdam 1100 DD Amsterdam, The Netherlands tel. 0031205668586 / fax. 0031205669573 info@skullbasecourse.nl m.b.vanhuiden@amc.uva.nl

esa

$a m$ 